\title{
Child Centred Care: Challenging Assumptions and Repositioning Children and Young People
}

\author{
Karen Ford ${ }^{\mathrm{a}, \mathrm{b}, *}$, , Annette Dickinson ${ }^{\mathrm{c}}$, Tineke Water ${ }^{\mathrm{c}}$, Steven Campbell ${ }^{\mathrm{d}}$, Lucy Bray ${ }^{\mathrm{e}}$, Bernie Carter ${ }^{\mathrm{e}}$ \\ ${ }^{a}$ Royal Hobart Hospital, Tasmania, Australia \\ b School of Health Sciences, University of Tasmania, PO Box 1061, Hobart, Tasmania 7000, Australia \\ c School of Clinical Sciences: Nursing, Auckland University of Technology, Private Bag 92006, Auckland 1142, New Zealand \\ d School of Health Sciences, University of Tasmania, Locked Bag 1322, Launceston, Tasmania. 7250, Australia \\ e Faculty of Health and Social Care, Edge Hill University, St Helens Road, Ormskirk, West Lancashire L39 4QP, United Kingdom
}

\section{A R T I C L E I N F O}

\section{Article history:}

Received 15 April 2018

Revised 16 August 2018

Accepted 18 August 2018

Available online $\mathrm{xxxx}$

\section{Keywords:}

Child-centred care

Family-centred care

Child

Health care

Ecological model

\begin{abstract}
A B S T R A C T
Theoretical Principles: Against a backdrop of increasing debate regarding children's voice and position within health care and the struggle to effectively implement Family-Centred Care (FCC) in practice, the concept of Child-Centred Care (CCC) has emerged.

Phenomena Addressed: The purpose of this paper is to explore the concept of CCC and its potential theoretical alignment with an ecological approach to health care.

Research Linkages: The paper will draw on practice-based research, highlighting the differences and similarities of CC against the more established FCC. Arguments will be presented to show that rather than competing with FCC, CCC has the potential to complement or extend traditional FCC, by placing children in a more prominent and central position than that which they currently hold within health care.
\end{abstract}

(c) 2018 Elsevier Inc. All rights reserved.

\section{Introduction}

The positioning of children in society is constantly evolving and being challenged and this debate has extended into discussion regarding the position of children within healthcare and the care models used. It has been proposed that the concept of child-centred care (CCC) may be one way of orientating children to a more central position within health care. The concept of CCC in health care is relatively new and, as Ford, Campbell, Carter, and Earwaker (2018) identify, not yet clearly defined. Therefore, it is timely to explore the potential philosophical basis for such an approach, how it might sit alongside more established models of care, and what it means for children, young people, families and health providers.

Child centred care forefronts children and young people, placing their interests at the centre of thinking and health care practice (Carter \& Ford, 2013). CCC acknowledges children and young people as agentic beings and social actors in their own right and promotes their right to be involved in their own health care (Carter, Bray, Dickinson, Edwards, \& Ford, 2014). Principles that underpin CCC include a holistic view of the child that sees them as more than just their illness or condition; concern for the overall experience of the child and family;

\footnotetext{
* Corresponding author at: Royal Hobart Hospital, Tasmania, Australia.

E-mail addresses: karen.ford@ths.tas.gov.au (K. Ford), Annette.dickinson@aut.ac.nz (A. Dickinson), Tineke.Water@aut.ac.nz (T. Water), Steven.Campbell@utas.edu.au (S. Campbell), Brayl@edgehill.ac.uk (L. Bray), Bernie.Carter@edgehill.ac.uk (B. Carter).
}

acknowledgement of children, young people and their parents as partners in care; advocacy for services to be co-ordinated around the child and family's needs; and ensuring appropriate transition to adult services (Department of Health, 2003). CCC presents nurses with a more radical way of thinking about how they include children, young people and families in care as the child's or young person's interests are seen as the starting point for care planning and provision.

The concept of CCC has gained momentum in the 21st century and references to child centred health services and CCC have become increasingly apparent in health literature and in shaping children's healthcare. Authors including Callery (2001), Glasper (2003) and Carter et al. (2014) identify the increased attention given to children's rights and interests which are inherent within CCC emanate, at least in part, as a direct result of the events leading up to the Bristol Royal Infirmary Inquiry and subsequent report (Kennedy, 2001). This inquiry found that some 30 to 35 deaths of children undergoing cardiac surgery were a result of 'flaws and failures... within the hospital, its organisation and culture' (Kennedy, 2001, p154) and that children's interests were subservient to systemic and institutional interests. This report found inadequacies at every point in children's chain of care, from referral to diagnosis, surgery and intensive care. Further, some staff lacked insight and their behaviour was flawed and there was a club culture and an environment where speaking out was not safe or acceptable. In Australia, the Garling (2008) Report into health services found similarly that children's rights and interests in health care are often given secondary consideration. This report found issues across a broad range of services, 
including (but not limited to) gross under-resourcing of mental health services for children and young people; uncertainty and indecision about where children should best receive care; problems in the transitioning of care from pediatric to adult services; fragmented services; and the (sometimes routine) co-location of adult patients with child patients that was risky and potentially harmful.

Although often claiming to be child-centred, the reality is that FCC can result in the child or young person having a more passive and less prominent role than that of their parent(s), even when their more active engagement should be possible. In CCC, the child comes first with the focus being on the child in the context of their family rather than the other way around (that is, where the focus tends to be on the parents with the child's perspectives being secondary) (Carter et al., 2014). Focusing heavily on the family as a main reference point for children, may fail to acknowledge the wider environment and relationships outside the family that children engage with. Therefore, it has been argued that the development of a new conceptual framework - CCC - is needed to elevate the child to a more prominent role as a key and active member of the partnership acknowledging the position of children within family but also beyond family relationships and environments. Bronfenbrenner's Ecological Model (Bronfenbrenner, 1986, 1999) places the child at the centre of the interactions between the people they engage with (for example, their friends, family members, health care professionals) and the immediate and wider environment, therefore provides a good fit with CCC as it goes beyond the focus of FCC on the child and family.

In this paper we explore the concept of CCC and the differences and similarities to the more familiar FCC. We highlight the conceptual fit with Bronfenbrenner's Ecological Model (Bronfenbrenner, 1986, 1999) and that of the United Nations Convention of the Rights of the Child (UNCRC), (United Nations General Assembly, 1989) and present some practical applications from our own research and practice. Our intention and our hope are to contribute to the discussion and to invite further comment.

\section{Child-centred Care an Ecological Approach}

We propose that CCC can be theoretically positioned within an ecological model and that an analysis from this perspective can assist understanding in regard to how CCC may be reflected in practice.

Bronfenbrenner's, 1979 monograph signalled the start of the development of an ecological model which placed the child at its centre (Bronfenbrenner, 1979). For Bronfenbrenner, children interact with multiple and changing environments over their lifetime and his theory provides an explanation of this interaction, and how these interactions influence growth and development. Through this theoretical lens, Bronfenbrenner emphasised the importance of understanding children in many environments and at different points in their lives, which he referred to as ecological systems. Bronfenbrenner's (1979) 'ecology' of childhood comprises five systems around the child starting with the one closest to the child. These systems are: The Microsystem, or the immediate environment; The Mesosystem which is about connection; The Exosystem or the indirect environment; the Macrosystem, the social and cultural values; and finally, the Chronosystem which allows for changes over time.

We believe an ecological view a provides a strong theoretical base for CCC and is consistent with more recent bio-developmental models which have drawn on ecological theory to acknowledge the impact that social and physical environments have on children's health (Shonkoff, 2010) and emphasise the importance of all relationships experienced by the child, not just those with parents (Kvalsvig, D'Souza, Duncanson, \& J., 2015) as positioned in models of FCC.

Importantly Bronfenbrenner's $(1986,1999)$ ecological model is also consistent with the United Nations Convention of the Rights of the Child (UNCRC), (United Nations Assembly, 1989) which acknowledges children as important members of society and explicitly states children's right to life, growth and development, alongside the right to make decisions about what matters to them. These two rights acknowledge the role of adults to ensure children's rights are met, alongside children as independent agents with subjective rights which they should be able to claim as their own (Liebel, 2017).

The benefit of using an ecological approach to view childhood, is that it can help health professionals to understand how a disruption in a child's environment caused by illness might impact on their wellbeing, growth and development as well as their ways of coping/adapting. How a child responds will be influenced by a range of factors such as age, the spheres of influence or support in each of the five systems, and the individual child. For example, a baby at the centre of these systems would be more reliant on the immediate microsystem provided by the primary caregiver (usually the mother), and less on the other four systems. If a baby becomes ill and requires hospitalisation, then child centred care is facilitated by supporting the mother to continue to provide that care. However, when a child goes to school, they gain greater independence from their parents and family, and the ecological systems of environment, connections and social and cultural values will widen. The reach of the disruption into school aged children's environments will be much broader if they have a long-term illness or hospitalisation. Not only do school aged children have meaningful connections with their parents, but also with others (such as other children/teachers) which may seem of less importance to the family and children's nurses. The strongest example of such disruption is for adolescents, who are much more likely to have strong connections with their peers and who assume central importance in an adolescents' immediate environment. Parents remain important (although this importance might not always be acknowledged by the adolescent) but they may have less of a central role in day-to-day lives than peers. The development of adolescent units (Bakke, 2016) in many hospitals has started the shift to placing the adolescent at the centre of care, rather than the parents. The usual development from neonate to adolescence is much more complex and dynamic than the three categories of baby, child and adolescence we have presented. However, these broad categories serve to remind health professionals to consider the development of capabilities as a continuum that are part of an ever-increasing widening ecological system.

It is also important to acknowledge that currently, a very western concept of childhood dominates thinking, therefore when using an ecological approach to CCC it is important to consider how other factors impact on childhood, for example, how specific societies view and construct childhood, including the specific culture and values, and the differences in childhood because of geography. For example, in Aoteaora/New Zealand (as in many 'global south' contexts) culturally Maori children exist in relationship not only to their parents, but also grandparents, extended family, ancestors and land (MOH, 1998) expressed within the holistic view of the four dimensions of health Te Whare Tapa Wha (Durie, 1994). Children are seen as the collective responsibility of the wider whanau (family) and hapu (community/ tribe) and these ties of kinship ensure the wellbeing of children who represent the future of the iwi (tribe) (https://teara.govt.nz). How society constructs childhood can also influence how children participate in all areas of their life. For example, much of how western (or global north) society constructs childhood reflects ideas around a child still 'becoming', not yet having the achieved the necessary competencies of adulthood, therefore needing adult protection. Liebel (2017) argues that this obliges children to see themselves through the eyes of those adults who have control over them and therefore may be unable to reject intervention in their lives because of dependency relationships. This may be in contrast to children brought up in different geographical locations such as in the global south, where there is more of an emphasis on shared responsibility in the family, with children participating in more tasks essential for their community and the separation between being a 'child' or 'adult' less obvious (Liebel, 2017).

All of these factors are important in considering the ecological environments children inhabit and the disruption that illness and 
hospitalisation may have on the child. The type of illness or disease will also exert different types of disruptions or responses. For example, a long-term illness may remain a persistent disruption throughout a child's life and what is 'normal' in their environment will likely be different than for children who experience a single episode of acute illness. A child with a long-term illness may be more dependent on parental support in many aspects of their life. Therefore, CCC calls nurses to focus on the child's environment, connections and cultural values and beliefs. Supporting children to reach their developmental potential remains the overall goal for all children and there is a need to acknowledge how this might be supported within health care settings, considering the individual and ecological environment.

Bronfenbrenner $(1986,1999)$ stresses that alongside understanding the ecological systems surrounding a child, it is also important to understand the individual child, including the personal characteristics, bioecological capacities and way in which the child systematically interacts with their environment. This includes firstly, a child's personal characteristics and dispositions, alongside those of the key people in a child's environment who influence the interactions between the child and their environment which in turn contributes to a child's development. Bronfenbrenner describes these forms of systematic interactions with a child's immediate environment as proximal processes which may either be preventative and protective. Issues can arise when these processes impede development, for example a lack of protection which can result in harm, or overly protective processes which impedes a child from participating in activities that may support their development. Secondly, each child has bioecological capacities, which includes abilities (current and future), experience, knowledge, and skill and which are the foundations of the good functioning of proximal processes during development. Thirdly, the way in which a child interacts with their environment, what the child expects from it, can develop or disrupt proximal processes, for example, trust in others around the child or previous experiences in certain situations/environments. The exertion of these factors (and the child's personal characteristics) alongside the influence of other people in their environment (parents teachers, health care professionals etc.) can influence how a child develops. When a child becomes unwell, the child's personal characteristics and ability to adapt to a changing environment may be challenged, adding to the pressure of dealing with an illness rather than normal development. Considering these characteristics, we believe, is foundational to a CCC approach.

A CCC approach requires the focus to be on supporting an individual child and their personal characteristics; acknowledging their existing ecological environments which are always evolving; and understanding the disruption an illness and hospitalisation may have on this. This calls for health care professionals to consider ways of placing the child in the centre of the environment to maintain a healthy and developing ecological system for them.

\section{Child-centred Care and Family-centred Care Different or Complementary?}

Despite the widely reported difficulties in regard to interpretation and implementation of FCC in practice, FCC is still frequently proposed as the most appropriate model for caring for children (Coyne, 2015). The question raised therefore is how does CCC differ and where does it fit in relation to FCC? The questions raised therefore are how CCC differs from or is complementary to the 175 well-established model of FCC?

Shields (2015) considers child centred care could be an alternative to FCC. This is also a view presented by Coyne, Hallström, and Söderbäck (2016) who suggest that CCC recognises children's rights to participation and decisions about their care and children's competence. Whilst involvement, participation and recognition of children's competence are certainly integral to CCC, we believe the link to competence may limit the dynamic nature and the prominence of the child in health care delivery. CCC proposes that the child's interests should have primary positioning in care irrespective of their age and ability (e.g. regardless of whether they are a premature infant, a young person transitioning to adult care or a child who is temporarily unable to voice their concern). It acknowledges that children's competence and participation will change over time and be influenced by their environment, culture and the previous positioning and actions of health care providers. It does not exclude the family but places the child as central to all care decisions and practices.

Foster (2015) proposes a model of 'family and child centred care (FCCC)' with the child coming after family (p5). Arguably, Foster's model places children's interests second to those of their family by not giving primary positioning to the child. If, as Foster seems to be suggesting FCC needs to integrate concepts of CCC, we respectfully suggest it be revised from FCCC to child and family centred care (CFCC) so that the child is positioned to come first rather than secondary to family.

It seems churlish, in many ways, to critique FCC as it certainly created a step change from the task- and clinically-oriented approaches to care delivery to children that preceded it. Despite its ambition and its pervasiveness as the approach that should underpin the care of children, FCC remains a 'partially mature and highly abstract concept' (Mikkelsen \& Frederiksen, 2011; p1152) and is subject to a growing number of critiques (Carter et al., 2014; Shields et al., 2012; Tallon, Kendall, \& Snider, 2015). These critiques are wide-ranging and include the lack of evidence for the effectiveness of FCC (Mikkelsen \& Frederiksen, 2011; Shields et al., 2012). Other critiques focus on the limitations imposed by the fact that FCC is based on attachment theory and that more contemporary models could provide a more robust, theoretically driven approach underpinned by a wide range of interdisciplinary knowledge (Tallon et al., 2015); our critique argues that Bronfenbenner's ecological model is a better starting point than attachment theory.

The evidence from the literature is that there is a considerable difference between what FCC should be and what actually occurs within practice settings (Khajeh, Dehghan Nayeri, Bahramnezhad, \& Sadat Hoseini, 2017). However, the fatal flaw that we and other authors perceive within the current framing and implementation of FCC is that all too frequently the focus is on collaboration between professionals and parents and the focus on the child is lost (Pritchard-Kennedy, 2012). This means that the child's voice is largely silent (Carter et al., 2014) and their subjectivity (Botbol, 2010) is overlooked. van Veelen et al. (2017, p232) note the tendency for professionals in child welfare settings to adopt "an adult point of view, omitting the child's perspective" and argue that addressing this requires envisioning and embedding different ways of working across all organisational levels; the same is true within health care.

Indeed, the most widely used measure of family-centred services, the MPOC-20 (Measure of Processes of Care), is focused entirely on the parent's experience of the processes of care. Despite this, many authors writing about the use of the MPOC-20 as a measure of FCC (Joachim, Wilk, Ryan, \& Speechley, 2016), fail to acknowledge that it is a proxy measure and seem not to notice that the perspectives of the child, who is supposedly at the core of the health care encounter, are not noted. As good as it is, the MPOC, is at best a parent-focused measurement of family-centeredness with one parent, usually the mother, acting as proxy for the 'family'. Findings from studies that do use the MPOC-20 show that, despite FCC underpinning many/most practice settings, practitioners are still not getting things comprehensively right for parents/families with the provision of general information being consistently rated as needing improvement (Cunningham \& Rosenbaum, 2014; Molinaro et al., 2017).

CCC has been described variously as an approach, a model or a way of thinking and there is some debate about just what it should be. Shields (2015), Coyne et al. (2016) and Carter et al. (2014) propose CCC as an alternative to FCC. Indeed, CCC might be an alternative and there is certainly strong evidence that FCC does not work terribly well 
in practice and that we fall short of addressing the problems associated with its implementation. However, we must take care that it is not a case of simply replacing one system that is not working very well with another potentially more challenging system. Importantly, CCC does not neglect the importance of family to the child's best interests. Indeed, it may often be in the child's best interests that we do practice FCC. Our view is that we do not think that it is a case of either /or but rather how do they come together.

\section{Tensions Inherent in Practice}

We do not suggest that using a CCC approach will be any less challenging than the difficulties encountered when implementing FCC. The following observation of a child participating in a study examining children's experience of clinical holding (Bray, Snodin, Carter, \& Twigg, 2013) when undergoing a clinical procedure illustrates some of the tensions faced when in practicing CCC.

A girl aged 4 walks into the phlebotomy room with her parents to have her bloods taken for the first time. She has had the 'magic' local anaesthetic cream on, and as she enters the room she is quiet and looks worried. Her dad sits down on the chair and puts the girl on his knee and cuddles her around her middle. The girl sees the equipment and starts saying 'no, no, no' and starts to cry, her dad directs the girl to look at her mum across the other side of the room and cuddles her more tightly. The health professional says, 'don't worry this will only take a minute' and takes and holds the girls' arm while her dad continues to cuddle her tightly so she cannot wriggle around. The girl is crying quite loudly when the butterfly is inserted. The procedure took about 30 seconds, a plaster is applied and she is released from the hold. The girl continues to be upset for several minutes afterwards and no-one in the room talks directly to her as she continues to sit on her dad's knee and the health professional sorts the bottles out. Mum then takes a magazine out of her bag and says 'look what I got you'.

Child-centred care, which advocates the fore-fronting of children's opinions and interests can be undermined when other people define and decide what is best for that child. In the above example the child's parents and health professional had decided that the child's best interests were served by getting the procedure completed as quickly as possible. The expressed wishes of the child to 'stop' or not start the procedure were over ruled by the adults present and the child's perspective was not sought; they were allocated a passive role. They were subsumed within their parents' (family) decision to hold them and proceed with the procedure. It could be argued that working within a FCC model diminishes the child's interests and their right to challenge, as the child's interests have been defined by their parents who are seen to know and act in their child's best interests.

If this case is considered within Bronfenbrenner's ecological model, the child (individual) is young, small and has little previous experience of health care procedures. They are dependent on the adults and the systems around them to forefront their rights and support their ability to participate in their health care. They need parents (microsystem) who are informed and can help them be actively involved, parents who know the ways to help their child through a procedure. They need health professionals (exosystem) who have the clinical time to spend with them and their parents to talk through what will happen and decide what strategies to use, who can ignore the busy waiting room and who can challenge the expectation that holding a child for a blood test is expected and acceptable practice. They need a health service which values long-term outcomes as well as short-term gains and targets. They need a society (macrosystem) which positions children as agentic beings and as having competency to join in conversations around what happens to them and their best interests. In order to move forward the concept and provision of CCC, the debate needs to be wider than CCC or FCC but needs to include a critical consideration of how a child's care is influenced, and in some cases constrained, by the wider context and environment.

\section{Conclusion}

We do not propose that the issues related to the practice of CCC are straight forward and, as illustrated above, these issues can be challenging. For example, if the child's interests are really primary, do we accept a parent's direction to just get on with a routine procedure or do we decide that the child's best interests override the need for the parent to not have to return to the hospital another day? Does taking this stance position nurses very differently than current practice? If we are truly nursing in a child centred way, does this require more courage and commitment? Can this approach actually work? There are no guarantees that this conceptual framework will work any better than FCC or any other models proposed in relation to the care of children in health care.

However, it is against this backdrop of FCC failing to adequately engage children in many of the everyday aspects of their health care and a strong predisposition to collaborate primarily with mothers, that we urge the need to rethink how we care for children. Authors whose focus is more firmly on the child's rights and willingness to be more fully engaged in their health care propose that it is time to more fully accept the child's centrality and are suggesting that health care for children becomes more person/child-centred (Gondek et al., 2017). Indeed, the shift within adult settings from a more clinically-driven model of care to a more person-centred approach accepts that the person/patient is part of a family and professionals need to consider the person within a complex set of relationships; 'person/patient and family-centred care' makes explicit in the name that both are important. Foster (2015) proposes we should move towards a 'family and child-centred' model of care arguing that it is more realistic to lead with the 'family' as parents have the responsibility to provide care for and promote their child's well-being. Whatever name (child-centred care, family-and childcentred care or child- and family-centred care) is given to the evolving approach to caring for children, we need to honour their right to be active participants and active partners along with their parents in their health care. We are not arguing against the importance of the child's family but are arguing for children to be able to play a fuller role, if they wish to do so. Achema and Ncama (2016) in a paper on FCC talk of the spirit of "ubuntu" as being central to care that is empathic and supportive. Although ubuntu is a contested word (Gade, 2012), it is popularly accepted as being a deeply rooted African word representing concepts of human interdependence and communalism that acknowledges the connections between people. Ubuntu is often summed up in statements such as "I am, because we are". Core to the concept is that people are not seen in isolation from each other. In exploring this concept further, we propose that "ubuntu" acknowledges that within healthcare people (child and parent and professionals) are in relationship with each other and with their environment. This aligns well with our ecological notion of child-centred care which recognises both the centrality of the child and the importance of their family, by acknowledging that 'a child is a child through their family'.

\section{Declaration of Interests}

None.

\section{Author Declaration}

The authors declare that this manuscript is original work and has not received prior publication or under consideration for publication elsewhere.

All authors contributed equally to the preparation of the manuscript and have read and reviewed each revision of the paper including the final paper submitted. 


\section{References}

Achema, G., \& Ncama, B. P. (2016). Exploring family-centered care for children living with HIV and AIDS in Nigeria. Japan Journal of Nursing Science, 13, 256-264. https://doi.org/ $10.1111 /$ jjns.12105.

Bakke, A. (2016). Empowering our youth: Initiating sexual health education on the inpatient unit for the chronically ill pediatric patient. Urologic Nursing, 36(6), 267-288. https://doi.org/10.7257/1053-816X.2016.36.6.267.

Botbol, M. (2010). Person-centered child and adolescent psychiatric care. International Journal of Integrated Care, 10(Suppl), e028.

Bray, L., Snodin, J., Carter, B., \& Twigg, E. (2013). Clinically holding children and young people for procedures or interventions within a children's hospital [Children's Research Fund project report.]. Edge Hill University.

Bronfenbrenner, U. (1979). The ecology of human development: Experiments in nature and design. Cambridge MA: Harvard University Press.

Bronfenbrenner, U. (1986). Ecology of the family as a context for human development: Research perspectives. Developmental Psychology, 22(6), 723.

Bronfenbrenner, U. (1999). Environments in developmental perspective: Theoretical and operational models. Measuring environment across the life span: Emerging methods and concepts (pp. 3-28). Washington DC: American Psychological Association.

Callery, P. (2001). Developing child-centred approaches to care. Neonatal, Paediatric and Child Health Nursing, 4(4), 3-4.

Carter, B., Bray, L., Dickinson, A., Edwards, M., \& Ford, K. (2014). Child-centred nursing: Promoting critical thinking. London: Sage.

Carter, B., \& Ford, K. (2013). Researching children's health experiences: The place for participatory, child-centered, arts-based approaches. Research in Nursing \& Health, 36(1), 95-107. https://doi.org/10.1002/nur.21517.

Coyne, I. (2015). Families and health-care professionals' perspectives and expectations of family-centred care: Hidden expectations and unclear roles. Health Expectations, 18 (5), 796-808. https://doi.org/10.1111/hex.12104.

Coyne, I., Hallström, I., \& Söderbäck, M. (2016). Reframing the focus from a family-centred to a child-centred care approach for children's healthcare. Journal of Child Health Care, 20(4), 494-502.

Cunningham, B. J., \& Rosenbaum, P. L. (2014). Measure of processes of care: A review of 20 years of research. Developmental Medicine and Child Neurology, 56(5), 445-452. https://doi.org/10.1111/dmcn.12347.

Department of Health (2003). Getting the right start: National service framework for children and young people: Standard for hospital services. Central Manchester and Manchester Children's University Hospital NHS Trust.

Durie, M. (1994). Whaiora: Maori health development. Oxford: Oxford Press.

Ford, K., Campbell, S., Carter, B., \& Earwaker, L. (2018). The concept of child-centered care in healthcare: A scoping review protocol. JBI Database of Systematic Reviews and Implementation Reports, 16(4), 845-851. https://doi.org/10.11124/jbisrir-2017003464.

Foster, M. (2015). Editorial: The family and child centred care model. Nursing Praxis in New Zealand, 31(3), 4-6.

Gade, C. B. N. (2012). What is Ubuntu? Different interpretations among South Africans of African descent. South African Journal of Philosophy, 31(3), 484-503.

Garling, P. (2008). Final report of the special commission of inquiry: Acute care services in NSW 352 public hospitals. 1. (pp. 353). Sydney: New South Wales Department of Premier and Cabinet, 353.

Glasper, E. A. (2003). Will they listen? Paediatric Nursing, 15(2), 3.

Gondek, D., Edbrooke-Childs, J., Velikonja, T., Chapman, L., Saunders, F., Hayes, D., \& Wolpert, M. (2017). Facilitators and barriers to person-centred care in child and young people mental health services: A systematic review. Clinical Psychology \& Psychotherapy, 24(4), 870-886. https://doi.org/10.1002/cpp.2052.

Joachim, K. C., Wilk, P., Ryan, B. L., \& Speechley, K. N. (2016). Family-centered care in children with epilepsy: Evaluating the measure of processes of care (MPOC-20). Epilepsia, 57(10), 1660-1668. https://doi.org/10.1111/epi.13494.

Kennedy, A. P. (2012). Systematic ethnography of school-age children with bleeding disorders and other chronic illnesses: Exploring children's perceptions of partnership roles in family-centred care of their chronic illness. Child: Care, Health and Development, 38(6), 863-869. https://doi.org/10.1111/j.1365-2214.2011.01310.x.

Kennedy, I. (2001). The report of the public inquiry into children's health surgery at Bristol royal infirmary 1984-1995: Learning from Bristol. London: Stationery Office.

Khajeh, M., Dehghan Nayeri, N., Bahramnezhad, F., \& Sadat Hoseini, A. S. (2017). Family centered care of hospitalized children: A hybrid concept analysis in Iran. Health Promotion Perspective, 7(4), 210-215. https://doi.org/10.15171/hpp.2017.37.

Kvalsvig, A., D'Souza, A., Duncanson, M., \& J., S. (2015). Pathways to child health, development and wellbeing: Optimal environments for orchids and dandelions. An overview of the evidence. Wellington: Ministry of Health.

Liebel, M. (2017). Children without childhood? Against the postcolonial capture of childhoods in the Global South. In A. Invernizzi, M. Liebel, M. B. \& R. Budde (Eds.), Children without childhood? Against the postcolonial capture of childhoods in the Global South (pp. 79-97) Retrieved from https://www.researchgate.net/publication/306081304_ Children_Without_Childhood_Against_the_Postcolonial_Capture_of_Childhoods_in the_Global_South Retrieved Apr 062018.

Mikkelsen, G., \& Frederiksen, K. (2011). Family-centred care of children in hospital - A concept analysis. Journal of Advanced Nursing, 67(5), 1152-1162. https://doi.org/10. 1111/j.1365-2648.2010.05574.x.

Ministry of Health (1998). Consent in child and youth health: Information for practitioners. Retrieved March 16, 2018, from https://www.health.govt.nz/publication/ consent-child-and-youth-health-information-practitioners.

Molinaro, A., Fedrizzi, E., Calza, S., Pagliano, E., Jessica, G., Fazzi, E., ... Brina, C. D. (2017). Family-centred care for children and young people with cerebral palsy: Results from an Italian multicenter observational study. Child: Care, Health and Development, 43(4), 588-597. https://doi.org/10.1111/cch.12449.

Shields, L. (2015). What is "family-centred care"? European Journal for Person Centered Healthcare, 3(2), 139-144.

Shields, L., Zhou, H., Pratt, J., Taylor, M., Hunter, J., \& Pascoe, E. (2012). Family-centred care for hospitalised children aged 0-12 years. Cochrane Database of Systematic Reviews, 10. https://doi.org/10.1002/14651858.CD004811.pub3.

Shonkoff, J. P. (2010). Building a new biodevelopmental framework to guide the future of early childhood policy. Child Development, 81(1), 357-367. https://doi.org/10.1111/j. 1467-8624.2009.01399.x.

Tallon, M. M., Kendall, G. E., \& Snider, P. D. (2015). Rethinking family-centred care for the child and family in hospital. Journal of Clinical Nursing, 24(9-10), 1426-1435. https:// doi.org/10.1111/jocn.12799.

United Nations General Assembly (1989). Adoption of a convention on the rights of the child. New York: United Nations.

van Veelen, J. S. M., Regeer, B. J., Broerse, J. E. W., van de Poel, S. F. P., \& Dinkgreve, M. A. H. M. (2017). Embedding the notion of child- and family-centered care into organizational practice: Learning from organizational visioning. Journal of Public Child Welfare, 11(2), 231-259. https://doi.org/10.1080/15548732.2016.1267068. 